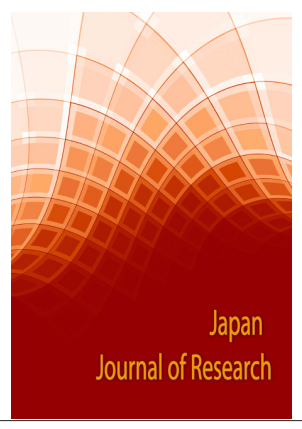

Correspondence

$\mathrm{Yi} \mathrm{Hu}$

Department of Oncology Medicine, Chinese PLA General Hospital, Beijing 100035, China E-mail: huyi301zlxb@sina.com

Jinfeng Li

Department of Oncology Medicine, Chinese PLA General Hospital, Beijing 100035, China E-mail: lijinfeng148@163.com।

Tel: +86-10-66937292

\footnotetext{
- Received Date: 23 Aug 2021

- Accepted Date: 30 Nov 2021

- Publication Date: 19 Dec 2021
}

Keywords

Compound Zhuye Shigao Granule, oral mucositis, chemotherapy, cancer, retrospective analysis

Copyright

(c) 2021 Science Excel. This is an openaccess article distributed under the terms of the Creative Commons Attribution 4.0 International license.

\title{
A retrospective analysis of the effects of CZSG on oral mucositis induced by chemotherapy in patients with malignant tumor
}

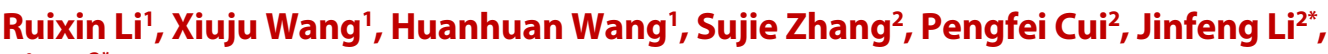 \\ $\mathrm{Yi} \mathrm{Hu}^{2 *}$ \\ ${ }^{1}$ Department of Oncology, First Department of Medical Oncology, Chinese PLA General Hospital , Beijing, China \\ ${ }^{2}$ Department of Oncology Medicine, Chinese PLA General Hospital, Beijing 100035, China
}

\begin{abstract}
The aim of this study was to quantitatively evaluate the effects of Compound Zhuye Shigao Granule (CZSG) on Oral mucositis (OM) induced by chemotherapy in patients with malignant tumor. The patients in CZSG group started taking CZSG one day before the next chemotherapy cycle (12g each time, 3 times a day for 10 days), the control group without any treatment before chemotherapy. Differences in the incidence and severity of OM between the two groups were examined statistically according to World Health Organization scale for OM. The pain sensation, xerostomia, and taste alteration were evaluated by the Common Terminology Criteria for Adverse Events v3.0 (CTCAE). And the median duration of $\mathrm{OM}$ were also recorded between the two groups. There was a significant difference in the OM incidence between the control group and the CZSG group $(P<0.01)$. All the accompanying symptom pain, xerostomia, and taste alteration differed between the two groups $(P<0.0001)$. And the median duration of OM was significantly reduced in the CZSG group as compared to the control group $(P<0.0001)$. Therefore our results show that CZSG has the advantage of rapid onset, good safety and few side effects in the prevention and treatment of OM induced by chemotherapy.
\end{abstract}

\section{Background}

Chemotherapy is one of the main approaches to treating malignant neoplasms [1,2]. The chemotherapy drugs, cisplatin, and methotrexate can be used to kill the tumor cells that reproduce rapidly, but these also affect the highly proliferative normal cells, such as hematopoietic systems, gastrointestinal and oral mucosal epithelial cells [2-4]. Oral mucositis (OM) is a common adverse effect of the chemotherapy, it results from direct toxic injury to the mucosal epithelial cells by the chemotherapy drugs. Lesions of the oral mucosa may inhibit liquid and solid food intake of cancer patients, resulting in lack of nutrition, water electrolyte disorders, weakened immunity, serious systemic infection and life-threatening, and it will eventually affect the overall effectiveness of anticancer therapy [57]. Therefore, it is particularly important to take effective preventive measures to prevent and reduce the occurrence of $\mathrm{OM}$.

This retrospectively study compared the incidence and severity of OM between a group of 64 patients who started taking Compound Zhuye Shigao Granule (CZSG) one day before chemotherapy (CZSG group) and a group of 85 patients who not taking CZSG (control group). We found that the incidence of $\mathrm{OM}$ in CZSG group significantly decreased than that of the control group. CZSG is a new drug self- developed by Dr Junzhang Lu in our hospital, a traditional Chinese medicine (TCM) compound based on the Zhuye gypsum soup, previous animal experiments and clinical studies have demonstrated CZSG has effective prevention and treatment of acute radiation esophagitis and recurrent oral ulcer $[8,9]$. The results of our study showed that CZSG also had a strong prevention and treatment effect on $\mathrm{OM}$ induced by chemotherapy, which provided a new clue for OM precaution in patients with malignant tumors.

\section{Methods}

Sample and setting

The Ethics Committee of the Chinese People's Liberation Army General Hospital approved this retrospective cohort study on the incidence and severity of OM of patients with liver cancer, gastric cancer, colon cancer and pancreatic cancer who were treated with chemotherapy admitted to the Cancer Center of the Chinese People's Liberation Army General Hospital from December 2017 to December 2019 (ethical approval number: 2017106). The enrollment of patients took place as soon as they developed OM after initiation of chemotherapy irrespective of chemotherapy stage. 149 patients were identified in this study. The patients in observation group started taking CZSG (Beijing Dongsheng Pharmaceutical Co., LTD. Batch number: 20160601, 12g/bag ) one day before 
the next chemotherapy cycle and $12 \mathrm{~g}$ each time, 3 times a day for 10 days, the control group without any treatment. Once OM occurred, patients in the control group gargled $10-15 \mathrm{ml} 1 \mathrm{mU} /$ $\mathrm{ml}$ Self-made Bacteromycin solution [10 bacteromycin tablets (Zhejiang Zhenyuan Pharmaceutical Co., LTD., Zhejiang, China, $50 \mathrm{mU} /$ tablet) were dissolved in $500 \mathrm{ml}$ bacteriostatic water for injection], 5 times a day after gargling with normal saline, and also gargled 10-15ml Kangfuxin Liquid (Sichuan Panxi Pharmaceutical Co., LTD., Sichuan, China), 5 times a day. Patients with severe pain were given $10-15 \mathrm{ml} 1 \mathrm{mg} / \mathrm{ml}$ lidocaine hydrochloride gargle (Suicheng Pharmaceutical Co., LTD., Tianjin, China).

Patients were excluded because of death or transferring to another hospital, serious organic lesions, autoimmune diseases, severe liver and kidney dysfunction, cachexia, and missing follow-up.

\section{Evaluation of $\mathrm{OM}$}

Differences in the incidence and severity of OM between the CZSG group and the control group were examined statistically according to World Health Organization (WHO) scale for OM [10]. Grade-0: none, Grade-I: Discomfort or pain and erythema of oral mucosa, Grade-II: Defects, ulcers or pseudomembranes, food accepted without limitations, Grade-III: Defects, ulcers or pseudomembranes, only liquids or creams accepted, Grade-IV: Defects, ulcers or pseudomembranes, not possible to eat food or liquids. The pain sensation, xerostomia, and taste alteration were evaluated by the Common Terminology Criteria for Adverse Events v3.0 (CTCAE) [11,12]. We also recorded the median duration of OM in the two groups. .

\section{Statistical analysis}

Statistical analyses were performed using Graphpad Prism 6.02. Parametric data were presented as means \pm SEM and tested using T-test. Categorical variables were analyzed by Chi-square test or Fisher's exact test. The nonparametric variables (median) were assessed by Mann-Whitney U-test. Relative risk and 95\% confidence intervals (CI) for OM, pain, xerostomia, and taste disturbance were determined. $P<0.05$ was considered statistically significant.

\section{Results}

\section{Patient characteristics}

149 patients with liver cancer, gastric cancer, colon cancer and pancreatic cancer were enrolled in our study. Of the 85 patients in the control group, 50 (58.8\%) were males and $35(41.2 \%)$ were females. Their ages ranged from 34 to 89 years, with a median age of 56.5 years. Of the 64 patients in the CZSG group, 35 (54.7\%) were males and $29(45.3 \%)$ were females. Their ages ranged from 33 to 85 years, with a median age of 58.3 years. The baseline patient characteristics were shown in Table 1 . There were no significant differences in gender, age and cancer type between the control group patients and the CZSG group patients.

Assessment of $\mathrm{OM}$ and the accompanying symptom

Table 2 depicted the grade of OM (as per WHO scale for OM) of all patients under the study. $11.7 \%$ grade- $0,14.1 \%$ grade-I, $30.6 \%$ grade-II, $20.0 \%$ grade-III and $23.6 \%$ grade-IV in the control group. $85.9 \%$ grade- $0,7.8 \%$ grade-I and $6.3 \%$ grade-II, remained in the CZSG group, whereas no grade-III and grade-IV patients were in the CZSG group. There was a significant difference in the

Table 1. Patient characteristics [n (\%)]

\begin{tabular}{|l|c|c|c|}
\hline \multicolumn{1}{|c|}{ Characteristic } & $\begin{array}{c}\text { Control group (\%) } \\
(\mathbf{n = 8 5 )}\end{array}$ & $\begin{array}{c}\text { CZSG group (\%) } \\
\text { (n=64) }\end{array}$ & p value \\
\hline Median age (years) & $56.5 \pm 10.3$ & $58.3 \pm 7.1$ & 0.470 \\
\hline Sex & & & 0.621 \\
Male & $50(58.8)$ & $35(54.7)$ & \\
Female & $35(41.2)$ & $29(45.3)$ & 0.804 \\
\hline lung cancer & 30 & 20 & \\
I & $4(13.3)$ & $1(5.0)$ & \\
II & $10(33.3)$ & $8(40.0)$ & 0.449 \\
III & $13(43.4)$ & $9(45.0)$ & \\
IV & $3(10.0)$ & $2(10.0)$ & \\
\hline gastric cancer & 20 & 16 & \\
I & $1(5.0)$ & 0 & \\
II & $8(40.0)$ & $9(56.2)$ & \\
III & $5(25.0)$ & $5(31.3)$ & \\
IV & $6(30.0)$ & $2(12.5)$ & \\
\hline ovarian cancer & 20 & 18 & \\
I & 0 & $1(5.6)$ & \\
II & $6(30.0)$ & $4(22.2)$ & \\
III & $8(40.0)$ & $8(44.4)$ & \\
IV & $6(30.0)$ & $5(27.8)$ & \\
\hline pancreatic cancer & & & \\
I & 15 & 10.644 \\
II & $4(26.7)$ & 0 & \\
III & $7(46.7)$ & $3(40.0)$ & \\
IV & $3(20.0)$ & $3(30.0)$ & \\
& & & \\
\hline
\end{tabular}


Table 2. The evaluation of $O M[n(\%)]$

\begin{tabular}{|c|c|c|c|c|}
\hline Grade & $\begin{array}{c}\text { Control group (\%) } \\
(\mathbf{n}=\mathbf{8 5})\end{array}$ & $\begin{array}{c}\text { CZSG group (\%) } \\
(\mathrm{n}=64)\end{array}$ & $\chi^{2}$ & $p$ \\
\hline $\begin{array}{l}\text { OM } \\
0 \\
\text { I } \\
\text { II } \\
\text { III } \\
\text { IV } \\
\text { All grade } \\
\end{array}$ & $\begin{array}{l}10(11.8) \\
12(14.1) \\
26(30.6) \\
17(20.0) \\
20(23.5) \\
75(88.2)\end{array}$ & $\begin{array}{c}55(85.9) \\
5(7.8) \\
4(6.3) \\
0 \\
0 \\
9(14.1)\end{array}$ & 85.92 & $<0.0001$ \\
\hline $\begin{array}{l}\text { Pain } \\
0 \\
\text { I } \\
\text { II } \\
\text { III } \\
\text { All grade } \\
\end{array}$ & $\begin{array}{l}12(14.1) \\
15(17.7) \\
36(42.3) \\
22(25.9) \\
73(85.9)\end{array}$ & $\begin{array}{c}55(85.9) \\
6(9.4) \\
3(4.7) \\
0 \\
9(14.1) \\
\end{array}$ & 80.01 & $<0.0001$ \\
\hline $\begin{array}{l}\text { Xerostomia } \\
0 \\
\text { I } \\
\text { II } \\
\text { III } \\
\text { All grade }\end{array}$ & $\begin{array}{l}10(11.8) \\
17(20.0) \\
36(42.3) \\
22(25.9) \\
73(88.2)\end{array}$ & $\begin{array}{c}56(87.5) \\
5(7.8) \\
3(4.7) \\
0 \\
9(12.5)\end{array}$ & 87.30 & $<0.0001$ \\
\hline $\begin{array}{l}\text { Taste alteration } \\
0 \\
\text { I } \\
\text { II } \\
\text { All grade }\end{array}$ & $\begin{array}{c}9(10.6) \\
47(55.3) \\
29(34.1) \\
76(89.4)\end{array}$ & $\begin{array}{c}45(70.3) \\
16(25.0) \\
3(4.7) \\
19(29.7)\end{array}$ & 58.58 & $<0.0001$ \\
\hline
\end{tabular}

Table 3. Comparison of duration for OM in control group and CZSG group

\begin{tabular}{|c|c|c|c|c|}
\hline $\begin{array}{c}\text { Duration of OM (in } \\
\text { days) }\end{array}$ & $\begin{array}{c}\text { Control group } \\
(\mathbf{n}=\mathbf{7 5})\end{array}$ & $\begin{array}{c}\text { CZSG group } \\
(\mathbf{n}=\mathbf{9})\end{array}$ & $\begin{array}{c}\text { Mann Whitney test } \\
\text { value, }\end{array}$ & $p$ \\
\hline Median (Range) & $10(6-15)$ & $6(3-8)$ & 4.5 & $<0.0001$ \\
\hline
\end{tabular}

OM incidence and severity between the control group and the CZSG group ( $\chi 2$ value $=85.92, P<0.0001)$. Pain, xerostomia, and taste alteration are usually the accompanying symptom of OM $[3,4]$. And all the accompanying symptom differed between the two groups $(P<0.0001)$. Table 3 showed that the median duration of OM was significantly reduced in the CZSG group [6(3-8)] as compared to the control group [10(6-15)] $(P<0.0001)$.

\section{Discussion}

$\mathrm{OM}$ is an inflammation and ulceration of the mucosal membranes in the mouth and throat, a common side effect and serious complication for patients receiving chemotherapy [5,13]. Most of patients will go for treatment when severe oral ulcer appeared after chemotherapy, while at this time OM worsens and can be difficult to manage [14]. Patients will experience severe pain and have difficulty in eating, seriously affecting their quality of life, and even causing sepsis due to bacterial invasion $[3,15]$. Therefore, the prevention of OM caused by chemotherapy is more important than the treatment. Because of the specificity of the chemotherapy cycle, it is possible to prevent OM caused by chemotherapy.

In March 2017, a lung cancer patient in our department developed grade IV OM 6 days after 4 chemotherapy cycles (Chemotherapy regimens: Albumin taxol and cisplatin), the ulcer in the mouth hadn't healed for one week after gargling nystine solution and Kangfu new solution. Then TCM doctors in our hospital advised the patient to take CZSG, and it was unexpected that this medicine worked quickly. Later, this patient took CZSG on his own initiative before the next chemotherapy cycle, to prevent recurrence of OM. And then we surprisingly found that this person didn't suffer from OM until the next cycle of chemotherapy was over. Nurses are often on the front lines of care and knowledge of cancer patients, proper care and management of these patients allow for better support. While as the conscientious contemporary nurses, our responsibility is not only to care for the patient, but also to communicate with the patients in order to better improve the quality of life of the patient. Therefore, we tried to suggest that patients with OM in last chemotherapy cycle begin to take CZSG one day before the next chemotherapy cycle, and whether OM happened or not the patients also took CZSG for 10 days. The medical staff found most patients also didn't develop OM in the next chemotherapy cycle same as the first patient. Therefore, we usually recommended that the attending physician gave the patient a doctor's advice to oral CZSG one day before chemotherapy, and with active publicity, education and care throughout the chemotherapy process. 
To prove the efficacy of CZSG, the incidence and severity of OM in cancer patients taking and not taking CZSG were retrospectively analyzed in this study. The results showed that the incidence of OM was relatively high $(88.2 \%)$ in control group patients during the next chemotherapy cycle, however the vast majority of patients who took CZSG in advance did not develop OM, and even if OM occurred, it was not very serious. The tendency of $\mathrm{OM}$ was the same as in control group and CZSG group in subsequent chemotherapy cycles, the results were omitted. Accompanying symptoms of OM e.g. pain, xerostomia, and taste alteration were also milder in CZSG group than the control group. Moreover, the median duration of OM was shorter in CZSG group due to the mildness of symptoms.

Based on similar clinical symptoms, like acute radioactive esophagitis and recurrent oral ulcer, chemotherapy induced-OM also belongs to the category of "oral sores" and "oral selection" in TCM. It is caused by hyperactivity of the heart, dampness of the liver and gallbladder, and dampness of the spleen and stomach. As a category of heat-clearing medicinal material, Professor Junzhang Lu has proved that CZSG can effectively prevent and treat acute radioactive esophagitis and recurrent oral ulcers $[8,9]$. CZSG was composed of Lophatherum, Gypsum, Radix Ginseng, Radix Ophiopogonis, Rhizoma Pinelliae, Liquorice, Rabdosia, Oldenlandia diffusa, Sculellaria Barbata, Semen Coicis, and Curcuma Zedoaria. CZSG has the effects of detoxification via expelling heat and relieving restlessness. Radix ginseng and Radix Ophiopogonis, the minister medicine, have effects of supplementing qi and yin. Rhizoma pinelliae as the assistant medicine has effects of regulating Wei, which can alleviate vomiting and nausea. Liquorice as the courier medicine has functions of nourishing Pi (Spleen) and Wei [8]. Therefore CZSG can dispell wind and heat from body and clearing the lung and moistening dryness. CZSG has been clearly demonstrated to prevent and treat OM induced by chemotherapy in our study. In addition, all patients did not have any adverse reactions during taking CZSG, and there were no abnormalities in blood routine, liver and kidney functions.

There is no simple universal method to prevent and treat OM induced by chemotherapy, and the treatment varies considerably among different institutions [16-19]. So, while many studies have been conducted worldwide to discover an effective method for the management of OM associated with chemotherapy, but still there is no specific treatment regimen available for the management of OM. In my study, a single dose of CZSG before each chemotherapy cycle reduced the incidence and severity of $\mathrm{OM}$, and the drug was generally well tolerated. Therefore, our results provide a new and perspective orientation for promoting malignant tumor patients rehabilitation and improving their quality of life.

However, the main limitation is that CZSG is a self-developed TCM in our hospital, so it's not universal. While our study gives a good indication that TCM usually plays a surprising role in some diseases, even in the treatment of the coronavirus disease (COVID-19). Therefore, the physicians can seek suitable TCM to assist the treatment of cancer patients receiving chemotherapy, and to prevent the occurrence of OM.

\section{Limitations}

The main limitation is that CZSG is a self-developed TCM in our hospital, so it's not universal. While our study gives a good indication that TCM usually plays a surprising role in some diseases, even in the treatment of the coronavirus disease (COVID-19). Therefore, the physicians can seek suitable TCM to assist the treatment of cancer patients receiving chemotherapy, and to prevent the occurrence of OM.

\section{Conclusions and clinical implications}

In the process of careful nursing, we find CZSG is a precious gift for cancer patients receiving chemotherapy. Although it has some limitations, we still introduce this self-developed TCM compound to the world, so that patients with tumor can have a better choice when they have OM during chemotherapy at least in China.

\section{Acknowledgements}

The authors wish to thank the other nurses participated in this study in our department.

\section{Author contributions}

Study conception and design: $\mathrm{Yi} \mathrm{Hu}$ and JinFeng Li; Data collection: Huanhuan Wang and Sujie Zhang; Data analysis and interpretation: Ruixin Li, Xiuju Wang and Pengfei Cui; Drafting of the article: Ruixin Li; Critical revision of the article: Jinfeng Li.

\section{Declaration of conflicting interests}

The authors declared no potential conflicts of interest with respect to the research, authorship, and/or publication of this article.

\section{References}

1. Aghamohamamdi A, Hosseinimehr SJ. Natural Products for Management of Oral Mucositis Induced by Radiotherapy and Chemotherapy. Integr Cancer Ther. 2016;15(1):60-68.

2. Shah CP, Shields CL, Shields JA. Chemotherapy for Malignant Intraocular Tumors. Dev Ophthalmol. 2016;55:337-343.

3. Chen SC. Oral Dysfunction in Patients With Head and Neck Cancer: A Systematic Review. J Nurs Res. 2019;27(6):e58.

4. Mougeot JC, Stevens CB, Morton DS, Brennan MT, Mougeot FB. Oral Microbiome and Cancer Therapy-Induced Oral Mucositis. J Natl Cancer Inst Monogr. 2019;2019(53):lgz002.

5. Daugèlaitė G, Užkuraitytė K, Jagelavičienė E, Filipauskas A. Prevention and Treatment of Chemotherapy and Radiotherapy Induced Oral Mucositis. Medicina (Kaunas). 2019;55(2):25.

6. Lalla RV, Brennan MT, Gordon SM, Sonis ST, Rosenthal DI, Keefe DM. Oral Mucositis Due to High-Dose Chemotherapy and/or Head and Neck Radiation Therapy. J Natl Cancer Inst Monogr. 2019;2019(53):lgz011.

7. Münstedt K, Männle $\mathrm{H}$. Using Bee Products for the Prevention and Treatment of Oral Mucositis Induced by Cancer Treatment. Molecules. 2019;24(17):3023.

8. Wang LJ, Lu JZ, Cai BN, Li MW, Qu BL. Effect of compound Zhuye Shigao Granule ( ) on acute radiation-induced esophagitis in cancer patients: A randomized controlled trial. Chin J Integr Med. 2017;23(2):98-104.

9. Lu JZ, Zhao L, Ren JP, Cao XT, Li HX, Zhao H. Preventive and therapeutic effects of Chinese herbal medicine Compound Zhuye Shigao Granule in rats with radiation-induced esophagitis. Zhong Xi Yi Jie He Xue Bao. 2011;9(4):435-441.

10. Pakravan F, Ghalayani P, Emami H, Isfahani MN, Noorshargh P. A novel formulation for radiotherapy-induced oral mucositis: Triamcinolone acetonide mucoadhesive film. J Res Med Sci. 2019;24:63.

11. Hurt CN, Jones S, Madden TA, et al. Recurrence of vulval intraepithelial neoplasia following treatment with cidofovir or imiquimod: results from a multicentre, randomised, phase II trial (RT3VIN). BJOG. 2018;125(9):1171-1177.

12. Zhang $\mathrm{S}$, Chen $\mathrm{Q}$, Wang $\mathrm{Q}$. The use of and adherence to CTCAE v3.0 in cancer clinical trial publications. Oncotarget. 2016;7(40):65577-65588.

13. Dos Reis PE, Ciol MA, de Melo NS, Figueiredo PT, Leite AF, Manzi Nde M. Chamomile infusion cryotherapy to prevent oral mucositis induced by chemotherapy: a pilot study. Support Care Cancer. 2016;24(10):4393-4398. 
14. Bennett M. Pain management for chemotherapy-induced oral mucositis. Nurs Child Young People. 2016;28:25-29.

15. Katagiri H, Fukui K, Nakamura K, Tanaka A. Systemic hematogenous dissemination of mouse oral candidiasis is induced by oral mucositis. Odontology. 2018;106(4):389-397. Singh R, Sharma S, Kaur S, Medhi B, Trehan A, Bijarania SK. Effectiveness of Topical Application of Honey on Oral Mucosa of Children for the Management of Oral Mucositis Associated with Chemotherapy. Indian J Pediatr. 2019;86(3):224-228.

16. Roldan CJ, Nouri K, Chai T, Huh B. Methylene Blue for the Treatment of Intractable Pain Associated with Oral Mucositis.
Pain Pract. 2017;17(8):1115-1121.

17. Kawashita Y, Koyama Y, Kurita H, et al. Effectiveness of a comprehensive oral management protocol for the prevention of severe oral mucositis in patients receiving radiotherapy with or without chemotherapy for oral cancer: a multicentre, phase II, randomized controlled trial. Int J Oral Maxillofac Surg. 2019;48(7):857-864.

18. Shumsky A, Bilan E, Sanz E, Petrovskiy F. Oncoxin nutritional supplement in the management of chemotherapy- and/or radiotherapy-associated oral mucositis. Mol Clin Oncol. 2019;10(4):463-468 